\title{
The magnetic moment of the Lee model
}

\author{
Miroslav Pardy \\ Department of Physical Electronics \\ Masaryk University, \\ Kotlářská 2, 61137 Brno, Czech Republic \\ e-mail:pamir@physics.muni.cz
}

November 20, 2019

\begin{abstract}
The Lee model of the unstable particle $V \rightleftharpoons N+\Theta$, where $\mathrm{N}$-particle is considered charged and $\Theta$-particle uncharged, is inserted into electromagnetic field. While the $\Theta$-particle propagates undisturbed, the N-particle is deflected by the extended photon source. The result of such process is the additional magnetic moment of the Lee particle. The Schwinger source theory is employed to present the calculation of the magnetic moment of the Lee model of the unstable particle.
\end{abstract}

\section{Introduction}

The Lee model of elementary particle was originally introduced by Lee (1954) as an exactly soluble and a renormalizable model that describes the interaction between pions and two neutral nucleons, where the nucleon can exist in two different intrinsic states. The particle corresponding to the Bose field is called $\Theta$ and the particles corresponding to the intrinsic states of the nucleon are called $V$ and $N$ particles.

The Lee unstable particle is defined by the equations

$$
V \rightleftharpoons N+\Theta,
$$


where $V, N, \Theta$ are particles, the physical characteristics of which fulfil some conservation laws.

We will suppose in this article that the particles participating in the process (1) have spin, mass and charge as follows:

$$
\Theta \equiv \Theta(0,0, \mu), \quad N \equiv N\left(\frac{1}{2}, e, m\right), \quad V \equiv V\left(\frac{1}{2}, e, \kappa\right) .
$$

Although this model is not realistic, the important features of nucleonpion system can be understood in a relatively simple way and one can get rid of the infinities without applying perturbation theory techniques. Moreover, the complete non-relativistic version of this model that describes one heavy particle sitting at some fixed point interacting with a field of non-relativistic bosons is as important as its relativistic counterpart.

\section{Source theory}

Source theory (Schwinger, 1970; Schwinger et al., 1976; Dittrich, 1978) was initially constructed for description of the particle physics situations occurring in the high-energy physics experiments. However, it was found that the original formulation simplifies the calculations in the electrodynamics and gravity where the interactions are mediated by photon, or, graviton respectively, where the source theory of gravity forms the analogue of quantum electrodynamics where in QED the interaction is mediated by the photon (with spin 1) and the gravitational interaction is mediated by the graviton (with spin 2) (Schwinger, 1970). The basic formula in the source theory is the vacuum-to-vacuum amplitude (Schwinger, 1970; Schwinger et al., 1976):

$$
\left\langle 0_{+} \mid 0_{-}\right\rangle=e^{\frac{i}{\hbar} W(S)},
$$

where the minus and plus tags on the vacuum symbol are causal labels, referring to any time before and after space-time region where sources are manipulated. The exponential form is introduced with regard to the existence of the physically independent experimental arrangements which has a simple consequence that the associated probability amplitudes multiply and corresponding $W$ expressions add (Schwinger, 1970; Schwinger et al., 1976). 
Source theory has a number of important advantages over standard quantum field theory. There are no ultraviolet divergences, no asymptotic states and no adiabatic hypothesis with the inevitable difficulties in adiabacity. The diagrams in this theory have immediate physical meaning.

The source theory version of quantum field theory enables to construct vacuum amplitudes and field equations for particles with arbitrary spins. The question arises how to extend the formalism in order to involve the specific experimental situation in particle physics. This situation is characterized by the phenomenological definition of the elementary particle. The elementary particle as such is defined only by the collision that create it. Although we do not know the details of how a particle is created, we recognize the production of the particle in a collision process as the result of a transfer of certain characteristic as energy, charge, spin etc. to the particle of interest. All the other particles are present to supply the net balance i.e. all the other particles act as the source of the particle of interest. They form so called the emission source.

We unite axiomatically the emission and the absorption sources under general heading of source $S(x)$ and this operation forms one of the postulates of the source theory.

Source $S(x)$ can be obviously defined indirectly through its effectiveness to create a particle similarly to the electric field which is defined how it acts on a charge. $S(x)$ is then a numerical measure that describes where the collision act occurs distributed in space-time.

The distribution of the collision process in momentum space is $S(p)$, and $S(x)$ and $S(p)$ are complementary aspects of the same source. They are related by a Fourier transform

$$
S(p)=\int(d x) S(x) e^{-i p x},
$$

where the notation and metrics was used as follows:

$$
\begin{gathered}
(d x)=d x^{0} d x^{1} d x^{2} d x^{3} ; \quad x=(c t, \mathbf{x})=\left(x^{0}, \mathbf{x}\right)=\left(-x_{0}, \mathbf{x}\right) \\
p x=\mathbf{p} \cdot \mathbf{x}-p^{0} x^{0} .
\end{gathered}
$$

We distinguish the strong sources and the weak ones. The strong source emits several particles during one act, while the weak source is defined as 
the source which creates only one particle.

Scalar particles are created by the scalar sources; vector, tensor and spinor particles are created by the vector, tensor and the spinor sources.

The neutral particles are described by the real sources, while charged particles are described by the complex sources.

Every collision process involves three elementary events. The emission of particle (or, particles), its propagation in space and the detection of the particle.

The vacuum amplitude ought to involve these particular subprocesses in order to describe the physical reality correctly. Let us show the decomposition of the vacuum amplitude in order to involve emission, propagation and detection of a particle.

$W(K)$ in $(3)$ is action which for the noninteracting spinless particles has the following form:

$$
W(K)=\frac{1}{2} \int(d x)\left(d x^{\prime}\right) K(x) \Delta_{+}\left(x-x^{\prime}\right) K\left(x^{\prime}\right),
$$

where $K(x)$ is the scalar source and $\Delta_{+}\left(x-x^{\prime}\right)$ is the propagation function which propagates the source effect from $x^{\prime}$ to $x$. It is symmetrical in $x$ and $x^{\prime}$, or,

$$
\Delta_{+}\left(x-x^{\prime}\right)=\Delta_{+}\left(x^{\prime}-x\right) .
$$

Now, let us consider the causal arrangement in which particles are created by source $K_{2}$, propagate in space-time and then are detected by source $K_{1}$ which is localized later in time than source $K_{2}$. Then, if we consider the case of the weak source, i.e. only one particle can be created or annihilated by a source, then, we write the vacuum persistence probability amplitude in the form:

$$
\left\langle 0_{+} \mid 0_{-}\right\rangle^{K}=\left\langle 0_{+} \mid 0_{-}\right\rangle^{K_{1}}\left\langle 0_{+} \mid 0_{-}\right\rangle^{K_{2}}+\sum_{p}\left\langle 0_{+} \mid 1_{p-}\right\rangle^{K_{1}}\left\langle 1_{p+} \mid 0_{-}\right\rangle^{K_{2}},
$$

where $\left\langle 1_{p_{+}} \mid 0_{-}\right\rangle^{K_{2}}$ is the probability amplitude of creation of the particle with momentum $p$ and $\left\langle 0_{+} \mid 1_{p_{-}}\right\rangle^{K_{1}}$ is the probability amplitude of annihilation of the particle with momentum $p$. We will show that the decomposition of the vacuum amplitude (8) is equivalent with the original amplitude (3) after suitable definition of the partial amplitudes in eq. (8) and accepting some postulates which are fundamental ingredients of the source theory. 
In the source theory only the total source

$$
K=K_{1}+K_{2}
$$

is of the physical significance which follows from the postulate of the spacetime conformity of sources (Schwinger, 1970). Then, the weak source limit of the vacuum persistence amplitude follows from formula (8) in the form:

$$
\begin{gathered}
\left\langle 0_{+} \mid 0_{-}\right\rangle^{K} \approx 1+\frac{i}{2} \int(d x)\left(d x^{\prime}\right) K_{1}(x) \Delta_{+}\left(x-x^{\prime}\right) K_{1}\left(x^{\prime}\right)+ \\
\frac{i}{2} \int(d x)\left(d x^{\prime}\right) K_{2}(x) \Delta_{+}\left(x-x^{\prime}\right) K_{2}\left(x^{\prime}\right)+ \\
i \frac{1}{2} \int(d x)\left(d x^{\prime}\right) K_{1}(x) \Delta_{+}\left(x-x^{\prime}\right) K_{2}\left(x^{\prime}\right) .
\end{gathered}
$$

For the weak source limit we can also write

$$
\left\langle 0_{+} \mid 0_{-}\right\rangle \approx 1+f(K) ; \quad|f(K)| \ll 1 ; \quad f(0)=0
$$

and introducing definitions

$$
\begin{gathered}
\left\langle 1_{p} \mid 0_{-}\right\rangle \stackrel{d}{=} i K_{p}=i \sqrt{d \omega_{p}} K(p) \\
\left\langle 0_{+} \mid 1_{p}\right\rangle \stackrel{d}{=} i K_{p}^{*}=i \sqrt{d \omega_{p}} K(-p),
\end{gathered}
$$

where $*$ denotes operation of complex conjugation, we have instead of eq. (10):

$$
\begin{gathered}
\left\langle 0_{+} \mid 0_{-}\right\rangle \approx 1+f\left(K_{1}\right)+f\left(K_{2}\right)+\sum_{p} i K_{p}^{*} i K_{p}= \\
1+f\left(K_{1}\right)+f\left(K_{2}\right)+\sum_{p} i \sqrt{d \omega_{p}} K_{1}(-p) i \sqrt{d \omega_{p}} K_{2}(p) .
\end{gathered}
$$

The quantity $d \omega_{p}$ is defined as

$$
d \omega_{p}=\frac{(d \mathbf{p})}{(2 \pi)^{3}} \frac{1}{2 p^{0}} ; \quad p^{0}=+\sqrt{\left(\mathbf{p}^{2}+m^{2}\right)}
$$

and it represents the invariant measure in momentum space. 


\section{Modified propagation function for scalar particles}

When a source emits enough energy to produce a particle, the twoparticle emission can occur and the two-particle exchange between sources. We will here assume the two-particle emission is caused by the interaction which can be described by the interaction Lagrangian. The mathematical structure of such Lagrangian depends on the physical model which is either realistic i.e. correctly reflecting the physical reality, or instructive, clarifying the substance of the theory.

In this section we will consider the instructive model with the interaction Lagrangian (Dittrich, 1974; 1978)

$$
\mathcal{L}_{i n t}=g \psi^{*} \psi \varphi,
$$

which replaces the more realistic Lagrangians. The symbol $g$ in eq. (1) is the coupling constant, field $\psi$ has mass $m, \mu$ is mass corresponding to the field $\varphi$ and sources corresponding to $\psi^{*}, \psi$ and $\varphi$ are $H^{*}, H$ and $K$.

It is obvious that the two-particle exchange between sources is the integral part of the one-particle exchange which leads to the modification of the propagation function (propagator) of the free particle. In our case we shall consider the modification of the $\mu$-particle propagator, assuming the two-particle exchange will be realized by $\psi$-particles. The two-particle system has mass that ranges from $2 m$ to $\infty$. Though we are here restricted to $\mathcal{L}_{\text {int }}$ given by eq.(16), the generalization for the different interactions can be obtained by some analogy.

The action corresponding to the scalar fields $\psi^{*}, \psi, \varphi$ with the interaction Lagrangian (16) is as follows:

$$
W=\int(d x)\left[K \varphi+H^{*} \psi+H \psi^{*}+\mathcal{L}\right],
$$

where

$$
\mathcal{L}=-\frac{1}{2}\left[(\partial \varphi)^{2}+\mu^{2} \varphi^{2}\right]-\left[\left(\partial \psi^{*}\right)(\partial \psi)+m^{2} \psi^{*} \psi\right]+g \psi^{*} \psi \varphi .
$$

The principle of the stationary action then implies the following set of the coupled field equations:

$$
\begin{gathered}
\left(-\partial^{2}+m^{2}\right) \psi(x)=H(x)+g \psi(x) \varphi(x) \\
\left(-\partial^{2}+m^{2}\right) \psi^{*}(x)=H(x)^{*}+g \psi^{*}(x) \varphi(x)
\end{gathered}
$$




$$
\left(-\partial^{2}+\mu^{2}\right) \varphi(x)=K(x)+g \psi^{*}(x) \varphi(x) .
$$

For $g=0$ we obtain the free theory, or in other words, the particles traveling without interaction.

Such situation is involved in the amplitude

$$
\begin{gathered}
\left\langle 0_{+} \mid 0_{-}\right\rangle^{K, H, H^{*}}=\left\langle 0_{+} \mid 0_{-}\right\rangle^{K}\left\langle 0_{+} \mid 0_{-}\right\rangle^{H, H^{*}}= \\
\exp \left\{\frac{i}{2} K \Delta_{+}^{\mu} K\right\} \exp \left\{i H^{*} \Delta_{+}^{m} H\right\} .
\end{gathered}
$$

Expanding eq. (22) in a series and adding the absorption and emission indexes 1 and 2 to the fields and sources, we can write:

$$
\left\langle 0_{+} \mid 0_{-}\right\rangle=-\int(d x)\left(d x^{\prime}\right) \psi_{1}^{*}(x) H_{2}(x) H_{2}^{*}\left(x^{\prime}\right) \psi_{1}\left(x^{\prime}\right),
$$

where the amplitude (23) corresponds to the situation where source $\mathrm{H}_{2} \mathrm{H}_{2}^{*}$ emits couple of particles with fields $\psi_{1}^{*}$ and $\psi_{1}$. The vacuum amplitude generated by expansion of $\exp \left\{(i / 2) \int(d x) \mathcal{L}_{\text {int }}\right\}$ is on the other hand equal to

$$
\left\langle 0_{+} \mid 0_{-}\right\rangle=i g \int(d x) \psi_{1}^{*}(x) \psi_{1}(x) \varphi_{2}(x) .
$$

After comparison of eq. (23) with eq. (24) we get the relation for so-called effective source $\mathrm{H}_{2} \mathrm{H}_{2}^{*}$ as follows:

$$
\left.i H_{2}(x) H_{2}^{*}\left(x^{\prime}\right)\right|_{\text {eff emiss }}=g \delta\left(x-x^{\prime}\right) \varphi_{2}(x) .
$$

We can picture the process of the two-particle emission by the effective source by means of the diagram. Circle represents there source, thin lines represent the causal propagation of real particles and the heavy line indicates the non-causal propagation of a virtual particle. Time is read vertically.

For the two-particle absorption, we get by the analogical procedure so called effective absorption source and its mathematical form is the following one (Dittrich, 1974; 1978):

$$
\left.i H_{1}(x) H_{1}^{*}\left(x^{\prime}\right)\right|_{\text {eff abs }}=g \delta\left(x-x^{\prime}\right) \varphi_{1}(x) .
$$

Unifying the process of emission and absorption, we get the real process consisting of the two-particle exchange between sources.

The two-particle exchange between effective sources is obviously involved in the vacuum amplitude 


$$
\left\langle 0_{+} \mid 0_{-}\right\rangle=\exp \left\{i \int H_{1}^{*} \Delta_{+}^{m} H_{2}\right\} \exp \left\{i \int H_{1} \Delta_{+}^{m} H_{2}^{*}\right\},
$$

because the following formula can be extracted from its expansion:

$$
\begin{gathered}
\left\langle 0_{+} \mid 0_{-}\right\rangle=\left.\int i H_{1}^{*}(x) H_{1}(y)\right|_{\text {eff abs }} \Delta_{+}^{m}\left(x-x^{\prime}\right) \quad \times \\
\left.\Delta_{+}^{m}\left(y-y^{\prime}\right) i H_{2}^{*}\left(x^{\prime}\right) H_{2}^{*}\left(y^{\prime}\right)\right|_{\text {eff emiss }},
\end{gathered}
$$

where we have introduced the effective sources.

Inserting relations (25) and (26) into eq. (28) we get the following mathematical form of the vacuum to vacuum amplitude for the two-particle exchange between effective sources:

$$
\left\langle 0_{+} \mid 0_{-}\right\rangle=g^{2} \int(d x)\left(d x^{\prime}\right) \varphi_{1}(x)\left[\Delta_{+}^{m}\left(x-x^{\prime}\right)\right]^{2} \varphi_{2}(x),
$$

which may be easily interpreted as the process of emission, propagation and absorption of the particle with mass $\mu$ by the field $\varphi$ acting here as a source.

For the causal arrangement of sources, i.e. $x^{0}>x^{\prime 0}$, we get:

$$
\begin{gathered}
{\left[\Delta_{+}^{m}\left(x-x^{\prime}\right)\right]^{2}=\left[\int i d \omega_{p} e^{i p\left(x-x^{\prime}\right)}\right]^{2}=-\int d \omega_{p} d \omega_{p^{\prime}} e^{i\left(p+p^{\prime}\right)\left(x-x^{\prime}\right)}=} \\
-\int d \omega_{p} d \omega_{p^{\prime}} e^{i k\left(x-x^{\prime}\right)}(2 \pi)^{3} \delta\left(p+p^{\prime}-k\right) \frac{(d k)}{(2 \pi)^{3}},
\end{gathered}
$$

where we have inserted the unit factor 1 represented as

$$
1=\int(2 \pi)^{3} \delta\left(p+p^{\prime}-k\right) \frac{(d k)}{(2 \pi)^{3}} .
$$

Supposing that $k$ is the four-momentum or the virtual photon, we have

$$
-k^{2}=M^{2}>(2 m)^{2}
$$

from which follows

$$
2 k^{0} d k^{0}=d M^{2}
$$

which gives

$$
\frac{(d k)}{(2 \pi)^{3}}=\frac{d \mathbf{k}}{(2 \pi)^{3}} d k^{0}=\frac{d \mathbf{k}}{(2 \pi)^{3}} \frac{1}{2 k^{0}} d M^{2}=d \omega_{k} d M^{2} .
$$


Using the last relations, we can write $\left(x^{0}>x^{0}\right)$ :

$$
\begin{gathered}
{\left[\Delta_{+}^{m}\left(x-x^{\prime}\right)\right]^{2}=i \int d M^{2} i d \omega_{k} e^{i k\left(x-x^{\prime}\right)} d \omega_{p} d \omega_{p^{\prime}}(2 \pi)^{3} \delta\left(p+p^{\prime}-k\right)=} \\
i \int d M^{2} \Delta_{+}\left(x-x^{\prime}, M^{2}\right) d \omega_{p} d \omega_{p^{\prime}}(2 \pi)^{3} \delta\left(p+p^{\prime}-k\right),
\end{gathered}
$$

where we have used relation

$$
i \int i d \omega_{k} e^{i k\left(x-x^{\prime}\right)}=\Delta_{+}\left(x-x^{\prime}, M^{2}\right) .
$$

The last integral in (35) is a function of $M^{2}$. In order to evaluate it, we have possibility to evaluate it in the rest frame of $k$, or, in other words for $k^{0}=0$. Then,

$$
\mathbf{p}+\mathbf{p}^{\prime}=0 ; \quad p^{0}=p^{0}=\sqrt{|\mathbf{p}|^{2}+m^{2}}=\frac{M}{2} ; \quad-k^{2}=M^{2} .
$$

Then, we get for this integral

$$
f\left(M^{2}\right)=\int d \omega_{p} d \omega_{p^{\prime}}(2 \pi)^{3} \delta\left(p+p^{\prime}-k\right)=\frac{1}{(4 \pi)^{2}}\left(1-\frac{4 m^{2}}{M^{2}}\right)^{1 / 2} .
$$

The formula (35) does not involve the mathematical procedure called the space-time extrapolation. This procedure involves the operational content consisting in replacing the $\Delta_{+}\left(x-x^{\prime}, M^{2}\right)$ in (35) by the modified function

$$
\Delta_{+}\left(x-x^{\prime}, M^{2}\right)=\int \frac{(d k)}{(2 \pi)^{4}} \frac{e^{i k\left(x-x^{\prime}\right)}}{k^{2}+M^{2}-i \varepsilon},
$$

where C. T. is so called contact term and it will be determined later. For the square $\left[\Delta_{+}^{m}\left(x-x^{\prime}\right)\right]^{2}$, we get:

$$
\left[\Delta_{+}^{m}\left(x-x^{\prime}\right)\right]^{2}=\frac{i}{(4 \pi)^{2}} \int\left[1-\left(\frac{2 m}{M}\right)^{2}\right]^{1 / 2} \Delta_{+}\left(x-x^{\prime}, M^{2}\right) d M^{2} .
$$

Finally, we get the vacuum-vacuum amplitude corresponding to the twoparticle exchange between sources in the following form transformed into the momentum space:

$$
\left\langle 0_{+} \mid 0_{-}\right\rangle=\frac{i}{(4 \pi)^{2}} g^{2} \quad \times
$$




$$
\int d M^{2} \sqrt{1-\frac{4 m^{2}}{M^{2}}} \frac{(d k)}{(2 \pi)^{4}} \varphi_{1}(-k)\left(\frac{1}{k^{2}+M^{2}-i \varepsilon}+\text { C.T. }\right) \varphi_{2}(k) .
$$

Using field source equations

$$
\begin{gathered}
\varphi_{2}(k)=\frac{1}{k^{2}+\mu^{2}-i \varepsilon} K_{2}(k) \\
\varphi_{1}(-k)=\frac{1}{k^{2}+\mu^{2}-i \varepsilon} K_{1}(-k)
\end{gathered}
$$

we get after insertion of $(42 \mathrm{a}, 42 \mathrm{~b})$ in the vacuum-vacuum amplitude (41)

$$
\begin{gathered}
\left\langle 0_{+} \mid 0_{-}\right\rangle=i \int \frac{(d k)}{(2 \pi)^{4}} K_{1}(-k) \times \\
\frac{g^{2}}{(4 \pi)^{2}} d M^{2} \sqrt{1-(2 m / M)^{2}}\left[\frac{1}{\left(k^{2}+\mu^{2}\right)^{2}} \frac{1}{k^{2}+M^{2}-i \varepsilon}\right] K_{2}(k),
\end{gathered}
$$

which indicates about double poles in $k^{2}=-\mu^{2}$. We shall see in the following text that the unacceptable poles can be suppressed by the suitable choosing of the contact term.

The vacuum amplitude for the free $\mu$-particle is

$$
\left\langle 0_{+} \mid 0_{-}\right\rangle^{(0)}=i \int \frac{(d k)}{(2 \pi)^{4}} K_{1}(-k) \Delta_{+}^{\mu}(k) K_{2}(k),
$$

which forms together with the amplitude (43) the total vacuum-to-vacuum amplitude involving the two-particle exchange between effective sources. The explicit form of this amplitude is as follows:

$$
\begin{gathered}
\left\langle 0_{+} \mid 0_{-}\right\rangle=i \int \frac{(d k)}{(2 \pi)^{4}} K_{1}(-k) \times \\
{\left[\Delta_{+}^{\mu}(k)+\frac{g^{2}}{(4 \pi)^{2}} \int d M^{2} I\left(M^{2}\right) \frac{1}{\left(k^{2}+\mu^{2}\right)^{2}} \frac{1}{k^{2}+M^{2}-i \varepsilon}\right] K_{2}(k),}
\end{gathered}
$$

where

$$
I\left(M^{2}\right)=\sqrt{1-\left(\frac{2 m}{M}\right)^{2}}
$$


From eq. (45) we can extract the total $\mu$-propagation function involving the two-particle exchange process in the form:

$$
\tilde{\Delta}_{+}^{\mu}(k)=\frac{1}{k^{2}+\mu^{2}-i \varepsilon}+\frac{1}{k^{2}+\mu^{2}-i \varepsilon} M\left(k^{2}\right) \frac{1}{k^{2}+\mu^{2}-i \varepsilon},
$$

where

$$
M\left(k^{2}\right)=\frac{g^{2}}{(4 \pi)^{2}} \int_{(2 m)^{2}}^{\infty} d M^{2} I\left(M^{2}\right)\left(\frac{1}{k^{2}+M^{2}-i \varepsilon}+C . T .\right) .
$$

As $M\left(k^{2}\right)$ does not vanish at $k^{2}=-\mu^{2}$ which means that the behavior of propagation function is drastically modified in neighborhood $k^{2}=$ $-\mu^{2}$. This contradicts the phenomenological basis of the theory. But on the other hand, the source couplings that are inferred through spacetime extrapolation of causal arrangement can always be supplemented by contact interactions. Unless additional physical considerations adduced, the contact terms can be omitted. In our case they play the role of suppressing the singularities in the neighborhood of $k^{2}=-\mu^{2}$. The natural requirement is

$$
M\left(k^{2}\right)=0 ; \quad \frac{\partial M\left(k^{2}\right)}{\partial k^{2}}=0 ; \quad \text { for } \quad k^{2}=-\mu^{2},
$$

which can be achieved by

$$
C . T .=-\frac{1}{M^{2}+\mu^{2}-i \varepsilon}+\left(\frac{k^{2}+\mu^{2}}{M^{2}-\mu^{2}}\right)^{2},
$$

which gives

$$
\begin{gathered}
M\left(k^{2}\right)=\left(k^{2}+\mu^{2}\right) \frac{g^{2}}{(4 \pi)^{2}} \times \\
\int_{(2 m)^{2}}^{\infty} d M^{2} I\left(M^{2}\right) \frac{1}{\left(M^{2}-\mu^{2}\right)^{2}} \frac{1}{k^{2}+M^{2}-i \varepsilon} .
\end{gathered}
$$

Then, the $\mu$-particle propagator function is of the form

$$
\begin{gathered}
\tilde{\Delta}_{+}^{\mu}(k)=\frac{1}{k^{2}+\mu^{2}-i \varepsilon}+ \\
\frac{g^{2}}{(4 \pi)^{2}} \int_{(2 m)^{2}}^{\infty} d M^{2} I\left(M^{2}\right) \frac{1}{\left(M^{2}-\mu^{2}\right)^{2}} \frac{1}{k^{2}+M^{2}-i \varepsilon},
\end{gathered}
$$

or, in the configuration space 


$$
\begin{gathered}
\tilde{\Delta}_{+}^{\mu}\left(x-x^{\prime}\right)=\Delta_{+}^{\mu}\left(x-x^{\prime}\right)+ \\
\frac{g^{2}}{(4 \pi)^{2}} \int_{(2 m)^{2}}^{\infty} d M^{2} I\left(M^{2}\right) \frac{1}{\left(M^{2}-\mu^{2}\right)^{2}} \Delta_{+}\left(x-x^{\prime} ; M^{2}\right) .
\end{gathered}
$$

\section{The Lee model in the electromagnetic field}

We follow here the article by author (Pardy, 1979) with the elimination of the typographical errors. So, we use the following notation for the corresponding fields, sources and the Green functions (propagators):

$$
\Theta \equiv \Theta\left(\varphi, J, \Delta_{+}\right), \quad N \equiv N\left(\psi, \eta, G_{+}\right), \quad V \equiv V\left(\chi, \zeta, G_{+}^{\kappa}\right) .
$$

The action $W$ in the fundamental source ansatz

$$
\left\langle 0_{+} \mid 0_{-}\right\rangle=e^{\frac{i}{\hbar} W(S)}
$$

is

$$
\begin{gathered}
W=W(\zeta, \eta, J)=\int(d x)\left\{\chi(x) \gamma^{0} \zeta(x)+\zeta(x) \gamma^{0} \chi(x)+\right. \\
\left.\psi(x) \gamma^{0} \eta(x)+\eta(x) \gamma^{0} \psi(x)+\varphi^{*}(x) J(x)+J^{*}(x) \varphi(x)+\mathcal{L}\right\},
\end{gathered}
$$

where

$$
\mathcal{L}=\mathcal{L}_{0}+\mathcal{L}_{\text {int }}
$$

and

$$
\begin{gathered}
\mathcal{L}_{0}=\chi \gamma^{0}\left(\gamma \frac{1}{i} \partial+\kappa\right) \chi+\psi \gamma^{0}\left(\gamma \frac{1}{i} \partial+m\right) \psi+\left(\partial \varphi^{*} \partial \varphi+\mu^{2} \varphi^{*} \varphi\right) \\
\mathcal{L}_{i n t}=-g\left(\psi \gamma^{0} \varphi \chi+\chi \gamma^{0} \varphi \psi\right)
\end{gathered}
$$

The differential equations for $\chi, \psi, \varphi$ follow from the variation of $W$. Their integral representations are as follows:

$$
\begin{aligned}
& \chi(x)=\int\left(d x^{\prime}\right) G_{+}^{\kappa}\left(x-x^{\prime}\right) \zeta\left(x^{\prime}\right) \\
& \psi(x)=\int\left(d x^{\prime}\right) G_{+}\left(x-x^{\prime}\right) \eta\left(x^{\prime}\right)
\end{aligned}
$$




$$
\varphi(x)=\int\left(d x^{\prime}\right) \Delta_{+}\left(x-x^{\prime}\right) J\left(x^{\prime}\right) .
$$

where the causal Green functions $G_{+}^{\kappa}, G_{+}, \Delta_{+}$obey equations

$$
\begin{aligned}
& \left(\gamma \frac{1}{i} \partial+\kappa\right) G_{+}^{\kappa}\left(x-x^{\prime}\right)=\delta\left(x-x^{\prime}\right) \\
& \left(\gamma \frac{1}{i} \partial+m\right) G_{+}\left(x-x^{\prime}\right)=\delta\left(x-x^{\prime}\right) \\
& \left(-\partial^{2}+\mu^{2}\right) \Delta_{+}\left(x-x^{\prime}\right)=\delta\left(x-x^{\prime}\right) .
\end{aligned}
$$

Now, we have in hands all the most important relations and we can approach derivation of the propagation function corresponding the process of emission and absorption (1).

\section{The V-particle propagation function}

The interaction term $\mathcal{L}_{\text {int }}$ involves the interaction which changes the motion of the free $\mathrm{V}$-particle and leads therefore to the modification of the Vpropagation function. The considered process includes the emission and the absorption. First, we will consider the emission.

\subsection{EMISSION}

The process

$$
V \rightarrow N+\Theta
$$

represents the emission of the $N$ and $\Theta$-particles by the $\mathrm{V}$-particle.

In the language of the source theory the process (62) has the following physical meaning: the extended $\mathrm{V}$-particle source created $N$ and $\theta$-particles by emitting the time-like momentum $P$.

The vacuum amplitude of the process (62) is

$$
\left\langle 0_{+} \mid 0_{-}\right\rangle^{\eta J}=\left\langle 0_{+} \mid 0_{-}\right\rangle^{\eta}\left\langle 0_{+} \mid 0_{-}\right\rangle^{J},
$$

where

$$
\begin{aligned}
& \left\langle 0_{+} \mid 0_{-}\right\rangle^{\eta}=e^{i \int(d x)\left(d x^{\prime}\right) \eta(x) \gamma^{0} G_{+}\left(x-x^{\prime}\right) \eta\left(x^{\prime}\right)} \\
& \left\langle 0_{+} \mid 0_{-}\right\rangle^{J}=e^{i \int(d x)\left(d x^{\prime}\right) J^{*}(x) \Delta_{+}\left(x-x^{\prime}\right) J\left(x^{\prime}\right)} .
\end{aligned}
$$


On the other hand, the first term in $\mathcal{L}_{\text {int }}$ describes of the emission of $\mathrm{N}$ and $\Theta$-particles. Therefore

$$
\left\langle 0_{+} \mid 0_{-}\right\rangle=e^{-i g \int(d x)\left(d x^{\prime}\right) \psi(x) \gamma^{0} \varphi^{*}(x) \chi\left(x^{\prime}\right)} .
$$

Expanding eqs. (63) and (66) gives for comparison:

$$
\left\langle 0_{+} \mid 0_{-}\right\rangle_{\text {emission }}=(-i)^{2} \int(d x)(d \xi) \psi(x) \gamma^{0} \eta(x) \varphi^{*}(\xi) J(\xi)
$$

and

$$
\left\langle 0_{+} \mid 0_{-}\right\rangle_{\text {emission }}=-i g \int(d x) \psi(x) \gamma^{0} \varphi^{*}(\xi) \chi(x) .
$$

After transition to the effective sources

$$
\left.J(\xi) \eta(x) \rightarrow J(\xi) \eta(x)\right|_{\text {eff }}
$$

and putting

$$
\left.i J(\xi) \eta(x) \rightarrow i J(\xi) \eta(x)\right|_{\text {eff.emission }}=-g \delta(x-\xi) \chi(x)
$$

we get the vacuum amplitudes (67) and (68) are equal. Obviously, the momentum representation of eq. (19) is as follows:

$$
\left.i J(k) \eta(p)\right|_{\text {eff.emission }}=-g \chi(P),
$$

where

$$
\begin{gathered}
\eta(p)=\int(d x) e^{-i p x} \eta(x) \\
J(k)=\int(d \xi) e^{-i k \xi} J(\xi) \\
P=p+k .
\end{gathered}
$$

In connection with eq. (74) we still define a quantity $M$ by relation:

$$
-P^{2}=M^{2}
$$

\subsection{ABSORPTION}

We mean the process

$$
N+\Theta \rightarrow V
$$

The second term in $\mathcal{L}_{\text {int }}$ 


$$
-g \int(d x) \chi(x) \gamma^{0} \varphi(x) \psi(x)
$$

involves the process (74). Thus we have to compare

$$
\left\langle 0_{+} \mid 0_{-}\right\rangle^{\eta J}=\left\langle 0_{+} \mid 0_{-}\right\rangle^{\eta}\left\langle 0_{+} \mid 0_{-}\right\rangle^{J}
$$

with

$$
\left\langle 0_{+} \mid 0_{-}\right\rangle=e^{-g \int(d x) \chi(x) \gamma^{0} \varphi(x) \psi(x)},
$$

or,

$$
\left\langle 0_{+} \mid 0_{-}\right\rangle_{\text {absorption }}=(-i)^{2} \int(d x)(d \xi) J^{*}(\xi) \eta(x) \gamma^{0} \varphi(\xi) \psi(\xi)
$$

and

$$
\left\langle 0_{+} \mid 0_{-}\right\rangle_{\text {absorption }}=-i g \int(d x) \chi(x) \gamma^{0} \varphi(x) \psi(x) .
$$

After transition to the effective sources

$$
\left.J^{*}(x) \eta(x) \gamma^{0} \rightarrow J^{*}(\xi) \eta(x) \gamma^{0}\right|_{e f f .}
$$

where we put

$$
\left.i J^{*}(\xi) \eta(x) \gamma^{0}\right|_{\text {eff.absorption }}=-g \delta(x-\xi) \chi(x) \gamma^{0}
$$

we get the vacuum amplitudes (80) and (81) are equal. Obviously, the momentum representation of eq. (70) is as follows:

$$
i J^{*}(-k) \eta(-p) \gamma^{0}=-g \chi(-P),
$$

Now, in view of the fact we are able to describe the process of emission and absorption, we can describe the total process (1) as it represents the causal arrangement of eq. (11) and eq. (25). The vacuum amplitude of such causal arrangement for $x^{0} \geq x^{0}, \xi^{0} \geq \xi^{\prime 0}$ is the following

$$
\begin{gathered}
\left.\left\langle 0_{+} \mid 0_{-}\right\rangle\right|_{\text {causal }}=\int(d x)\left(d x^{\prime}\right)(d \xi)\left(d \xi^{\prime}\right) \times \\
{\left[i J(\xi) \eta(x) \gamma^{0}\right]_{\text {eff.abs. }} G_{+}\left(x-x^{\prime}\right) \Delta_{+}\left(x-x^{\prime}\right)\left[i J\left(\xi^{\prime}\right) \eta\left(x^{\prime}\right) \gamma^{0}\right]_{\text {eff.emiss }}}
\end{gathered}
$$

Or, using eq.(70) and eq. (83), we have

$$
\left.\left\langle 0_{+} \mid 0_{-}\right\rangle\right|_{\text {causal }}=g^{2} \int(d x)\left(d x^{\prime}\right) \chi(x) \gamma^{0} G_{+}\left(x-x^{\prime}\right) \Delta_{+}\left(x-x^{\prime}\right) \chi\left(x^{\prime}\right) .
$$


At this stage, we can approach the determination of the causal vacuum amplitude for the $\mathrm{V}$-particle moving in the electromagnetic field.

\section{The V-particle in the electromagnetic field}

If electromagnetic field is present, the following transformations of the Green functions and wave function must be used:

$$
\begin{gathered}
G_{+}\left(x-x^{\prime}\right) \rightarrow G_{+}^{A}\left(x-x^{\prime}\right) \\
\Delta_{+}\left(x-x^{\prime}\right) \rightarrow \Delta_{+}^{A}\left(x-x^{\prime}\right) \\
\chi\left(x-x^{\prime}\right) \rightarrow \chi_{+}^{A}\left(x-x^{\prime}\right) .
\end{gathered}
$$

However, $\Delta_{+}\left(x-x^{\prime}\right)=\Delta_{+}^{A}\left(x-x^{\prime}\right)$ because $\Theta$-particle is neutral, So, we have for the vacuum amplitude (86) the formula:

$$
\left\langle 0_{+} \mid 0_{-}\right\rangle \rightarrow=g^{2} \int(d x)\left(d x^{\prime}\right) \chi^{A}(x) \gamma^{0} G_{+}^{A}\left(x-x^{\prime}\right) \Delta_{+}\left(x-x^{\prime}\right) \chi^{A}\left(x^{\prime}\right),
$$

where $G_{+}^{A}\left(x-x^{\prime}\right)$ is given by the formula (Schwinger, 1973)

$$
\begin{gathered}
G_{+}^{A}\left(x-x^{\prime}\right)= \\
\int \frac{(d p)}{(2 \pi)^{4}} e^{i e q \int_{x^{\prime}}^{x} d \xi^{\mu} A_{\mu}(\xi)} e^{i p\left(x-x^{\prime}\right)}(m-\gamma p) \frac{1}{p^{2}+m^{2}-e q \sigma F-i \varepsilon} .
\end{gathered}
$$

Let us remark that from relation (89) follows, that the Green function of a charged particle in electromagnetic field is formed from the Green function of the free particle by multiplying the latter by the factor

$$
\Phi=e^{i e q \int_{x^{\prime}}^{x} d \xi^{\mu} A_{\mu}(\xi)}
$$

and the transformations

$$
m^{2} \rightarrow m^{2}-\delta ; \quad \delta=e q \sigma F ; \quad \sigma F=\frac{1}{2} \sigma^{\mu \nu} F_{\mu \nu} .
$$




\section{Determination of $G_{+}\left(x-x^{\prime}\right) \Delta_{+}\left(x-x^{\prime}\right)$}

We shall use this fact in determination of $G_{+}^{A}\left(x-x^{\prime}\right) \Delta_{+}\left(x-x^{\prime}\right)$ in eq. (88). For $x^{0} \geq x^{\prime 0}$ we have (Schwinger, 1973):

$$
\begin{gathered}
\Delta_{+}\left(x-x^{\prime}\right)=i \int d \omega_{k} e^{i k\left(x-x^{\prime}\right)} \\
G\left(x-x^{\prime}\right)=i \int d \omega_{p} e^{i p\left(x-x^{\prime}\right)}(m-\gamma p),
\end{gathered}
$$

where

$$
\begin{aligned}
& d \omega_{k}=\frac{(d \mathbf{k})}{(2 \pi)^{3}} \frac{1}{2 k^{0}} \\
& d \omega_{p}=\frac{(d \mathbf{p})}{(2 \pi)^{3}} \frac{1}{2 p^{0}} .
\end{aligned}
$$

Eqs. (82) and (83) give

$$
G_{+}\left(x-x^{\prime}\right) \Delta_{+}\left(x-x^{\prime}\right)=(i)^{2} \int d \omega_{p} d \omega_{k} e^{i(p+k)\left(x-x^{\prime}\right)}(m-\gamma p) .
$$

From the spectral requirements

$$
-P^{2}=M^{2}, \quad P=p+k,
$$

we have for the three-dimensional momentum

$$
d\left(P^{0}\right)^{2}=-d M^{2}
$$

and

$$
\frac{(d P)}{(2 \pi)^{3}}=d \omega_{p} d M^{2}
$$

Using identity

$$
1=\int(2 \pi)^{3} \delta(P-p-k) \frac{(d P)}{(2 \pi)^{3}}=\int d \omega_{P} d M^{2}(2 \pi)^{3} \delta(P-p-k)
$$

we get

$$
\begin{gathered}
G_{+}\left(x-x^{\prime}\right) \Delta_{+}\left(x-x^{\prime}\right)= \\
\int d \omega_{P} d \omega_{k} d M^{2}(2 \pi)^{3} \delta(P-p-k) e^{i(P+k)\left(x-x^{\prime}\right)}(m-\gamma p) .
\end{gathered}
$$


After performing $k$ and $p$ integration in the last formula, we have:

$$
G_{+}\left(x-x^{\prime}\right) \Delta_{+}\left(x-x^{\prime}\right)=\int d \omega_{P} d M^{2} e^{i P\left(x-x^{\prime}\right)} F(P),
$$

where

$$
\begin{gathered}
F(P)=\frac{1}{(2 \pi)^{2}}\left(m-\frac{M^{2}+m^{2}-\mu^{2}}{2 M^{2}} \gamma P\right) \times \\
{\left[1-\frac{(m+\mu)^{2}}{M^{2}}\right]^{1 / 2}\left[1-\frac{(m-\mu)^{2}}{M^{2}}\right]^{1 / 2} .}
\end{gathered}
$$

\section{The space-time extrapolation and determination of $G_{+}^{A}\left(x-x^{\prime}\right) \Delta_{+}\left(x-x^{\prime}\right)$}

During the derivation of $G_{+}\left(x-x^{\prime}\right) \Delta_{+}\left(x-x^{\prime}\right)$ it was assumed that $x^{0} \geq x^{\prime 0}$ i.e. the detection source was in time later than the emission one. The generalization of this assumption was enabled the source theoretical axiom called space-time extrapolation (Schwinger, 1973). We mean by the spacetime extrapolation the transformation

$$
\int d \omega_{p} d \omega_{k} e^{i P\left(x-x^{\prime}\right)} \rightarrow \Delta_{+}\left(x, x^{\prime}, M^{2}\right)
$$

where

$$
\Delta_{+}\left(x, x^{\prime}, M^{2}\right)=\int \frac{(d P)}{(2 \pi)^{4}} e^{i P\left(x-x^{\prime}\right)} \frac{1}{P^{2}+M^{2}-i \varepsilon} .
$$

After insertion of eq. (103) in to eq. (101), we have

$$
G_{+}\left(x-x^{\prime}\right) \Delta_{+}\left(x-x^{\prime}\right) \rightarrow \int d M^{2} F(P) \Delta_{+}\left(x, x^{\prime}, M^{2}\right),
$$

where $F(P)$ is given by eq. (102). As we have remarked in section 4 . we can obtain the product $G_{+}^{A}\left(x-x^{\prime}\right) \Delta_{+}\left(x-x^{\prime}\right)$, which involves the electromagnetic field, from the field product $G_{+}\left(x-x^{\prime}\right) \Delta_{+}\left(x-x^{\prime}\right)$ by multiplication by the factor

$$
\Phi=e^{i e q \varphi\left(x, x^{\prime}\right)}
$$

and using transformation

$$
m^{2} \rightarrow m^{2}-\delta ; \quad \delta=e q \sigma F ; \quad \sigma F=\frac{1}{2} \sigma^{\mu \nu} F_{\mu \nu}
$$


in $G_{+}\left(x-x^{\prime}\right) \Delta_{+}\left(x-x^{\prime}\right)$. In such a way we have:

$$
\begin{gathered}
G_{+}^{A}\left(x-x^{\prime}\right) \Delta_{+}\left(x-x^{\prime}\right)= \\
\left.\Phi \int d M^{2} F(P) \Delta_{+}\left(x, x^{\prime}, M^{2}\right)\right|_{m^{2} \rightarrow m^{2}-\delta, M^{2} \rightarrow M^{2}-\delta}
\end{gathered}
$$

and after modification

$$
\begin{gathered}
G_{+}^{A}\left(x-x^{\prime}, m^{2}\right) \Delta_{+}\left(x-x^{\prime}, \mu^{2}\right) \rightarrow \\
\rightarrow \Phi \int d M^{2}\left\{\left(m-\frac{M^{2}+\sqrt{\alpha \beta}}{2 M^{2}} \gamma \Pi\right)+\frac{M^{2}+\sqrt{\alpha \beta}}{2 M^{4}} \gamma \Pi \delta\right\} \\
\times\left(I(0)+I^{\prime}(0) \delta\right)\left(\Delta_{+}\left(x-x^{\prime}, M^{2}-\delta\right),\right.
\end{gathered}
$$

where

$$
\begin{gathered}
\alpha=(m+\mu)^{2} ; \quad \beta=(m-\mu)^{2} \\
I=\left(\frac{1}{4 \pi}\right)^{2}\left[1-\frac{\alpha-\delta}{M^{2}-\delta}\right]^{1 / 2}\left[1-\frac{\beta-\delta}{M^{2}-\delta}\right]^{1 / 2} \\
I(0)=\left.I\right|_{\delta \rightarrow 0} ; \quad I^{\prime}(0)=\left.\frac{d I}{d \delta}\right|_{\delta \rightarrow 0} .
\end{gathered}
$$

In the presence of electromagnetic field we put

$$
\gamma P \rightarrow \gamma \Pi, \quad \Pi=p-e q A
$$

and using relation

$$
\Phi \Delta_{+}\left(x-x^{\prime}, M^{2}-\delta\right) \rightarrow \frac{1}{-(\gamma \Pi)^{2}+M^{2}-i \varepsilon}
$$

we have to the first power of $\delta$

$$
\begin{gathered}
G_{+}^{A}\left(x-x^{\prime}\right) \Delta_{+}\left(x-x^{\prime}\right) \rightarrow \\
\int d M^{2}\left[m-\frac{M^{2}+\sqrt{\alpha \beta}}{2 M^{2}} \gamma \Pi\right] I(0) \frac{1}{-(\gamma \Pi)^{2}+M^{2}-i \varepsilon}+ \\
\int d M^{2}\left[m-\frac{M^{2}+\sqrt{\alpha \beta}}{2 M^{2}}\right] I(0) \gamma \Pi \delta \frac{1}{-(\gamma \Pi)^{2}+M^{2}-i \varepsilon}
\end{gathered}
$$




$$
\int d M^{2}\left[\frac{M^{2}+\sqrt{\alpha \beta}}{2 M^{4}}\right] I(0) \gamma \Pi \delta \frac{1}{-(\gamma \Pi)^{2}+M^{2}-i \varepsilon}+C . T .
$$

where at this stage we have introduced the so called contact term C.T. It guarantees the nonsingular behavior propagation function at point $\gamma \Pi=-\kappa$.

The conditions, which determine the contact terms are as follows:

$$
\begin{gathered}
L(\gamma \Pi=-\kappa)=0 \\
\frac{d L}{(\gamma \Pi)}(\gamma \Pi=-\kappa)=0,
\end{gathered}
$$

where $L$ is the expression between $\chi(x) \gamma^{0} \chi$. The condition (116) and (117) can be realized by the following transformations:

$$
\begin{aligned}
\frac{1}{\gamma \Pi-M-i \varepsilon} & \rightarrow \frac{(\gamma \Pi+\kappa)^{2}}{(M-\kappa)} \frac{1}{\gamma \Pi-M-i \varepsilon} \\
\frac{1}{\gamma \Pi+M-i \varepsilon} & \rightarrow \frac{(\gamma \Pi+\kappa)^{2}}{(M-\kappa)} \frac{1}{\gamma \Pi+M-i \varepsilon} .
\end{aligned}
$$

Using the procedures (118) and (119), we have for the integral in (115):

$$
\begin{gathered}
-(\gamma \Pi+\kappa)^{2} g^{2} \int_{m+\mu}^{\infty} \frac{d M}{M}\left\{\frac{(M+m)^{2}-\mu^{2}}{(M-\kappa)} \frac{1}{\gamma \Pi+M-i \varepsilon}-\right. \\
\left.-\frac{(M-m)^{2}}{(M+\kappa)} \frac{1}{\gamma \Pi-M-i \varepsilon}\right\} I(0) .
\end{gathered}
$$

The last formula is at the limit $\gamma \Pi \rightarrow \gamma P$ the propagation function derived by Dittrich (1974).

\section{The magnetic moment of the Lee model}

The additional action term replacing

$$
\int(d x)\left(d x^{\prime}\right) \chi(x) \gamma^{0} M\left(x, x^{\prime}\right) \chi\left(x^{\prime}\right)
$$

is in the presence of electromagnetic field, the following one:

$$
g^{2} \int(d x)\left(d x^{\prime}\right) \chi(x) \gamma^{0} M\left(x, x^{\prime}, F\right) \chi\left(x^{\prime}\right),
$$


where $M(F)$ is given by eq. (115). This supplements the initial action expression

$$
\int(d x) \chi(x) \gamma^{0}(\gamma \Pi+\kappa) \chi\left(x^{\prime}\right) .
$$

In the presence of electromagnetic field, we have

$$
(\gamma \Pi+\kappa)=0,
$$

or,

$$
\gamma \Pi=-\kappa
$$

Then,

$$
\begin{gathered}
\left.M(F)\right|_{\gamma \Pi=-\kappa}=\int_{\alpha}^{\infty} d M^{2} \\
\left\{\left[m+\frac{M^{2}+\sqrt{\alpha \beta}}{2 M^{2}} \kappa\right] I^{\prime}(0) \frac{1}{M^{2}-\kappa^{2}}-\frac{M^{2}-\sqrt{\alpha \beta}}{2 M^{4}} I(0) \frac{\kappa}{M^{2}-\kappa^{2}}\right\} \delta
\end{gathered}
$$

Further we have

$$
g^{2} M(F)=\lambda \frac{e q \sigma F}{2 \kappa},
$$

where, (if we put $M^{2}=x$ )

$$
\begin{gathered}
\lambda=\frac{2 \kappa}{(4 \pi)^{2}} g^{2} \int_{\alpha}^{\infty} d x\left\{\left[m+\frac{x+\sqrt{\alpha \beta}}{2 x} \kappa\right] \frac{1}{x^{2}}[(x-\alpha)(x-\beta)]^{1 / 2} \frac{1}{x-\kappa^{2}}-\right. \\
\left.-\left[\frac{x+\sqrt{\alpha \beta}}{2 x^{2}}\right] \frac{1}{x}[(x-\alpha)(x-\beta)]^{1 / 2} \frac{\kappa}{x-\kappa^{2}}\right\} .
\end{gathered}
$$

The effective action is equal as it follows:

$$
\int(d x)\left(d x^{\prime}\right) \chi(x) \gamma^{0}\left(\gamma \Pi+\kappa+\lambda \frac{e q \sigma F}{2 \kappa}\right) \chi\left(x^{\prime}\right),
$$

where

$$
\lambda \frac{e q \sigma F}{2 \kappa}
$$

is the additional magnetic moment of the Lee model of elementary particle. For small $\alpha$ we can put approximately 


$$
[(x-\alpha)(x-\beta)]^{1 / 2} \approx x
$$

and we have from eq. (79) after elementary integration

$$
\lambda=\frac{2 g^{2}}{(4 \pi)^{2}}\left\{\left(\frac{\sqrt{\alpha \beta}}{\kappa^{2}}+\frac{m}{\kappa}\right) \lg \frac{\alpha}{\alpha-\kappa^{2}}-\frac{\sqrt{\alpha \beta}}{\alpha}\right\} .
$$

\section{Discussion}

In this paper, we have considered the relativistic Lee model in the framework of the Schwinger source theory of elementary particles. After inserting the Lee model into the constant electromagnetic field we observe new term in the Lee model which corresponds to the magnetic moment of the Lee unstable particle.

References

Lee T. D. (1954). Some Special Examples in Renormalizable Field Theory, Phys. Rev. 95, 1329.

Dittrich. W. (1974). Lee model and source theory: A new method of calculation, Phys. Rev. D 10, 1902

Dittrich, W. (1978). Source Methods in Quantum Field Theory, Fortschr. Phys. 26, 289.

Pardy, M. (1979). The Lee model in electromagnetic field, Scripta Fac. Sci. UJEP Brunensis, Physica 1, 9, 41-48.

Schwinger, J., Tsai, W. Y., and Erber, T. (1976). Classical and quantum of synergic synchrotron Čerenkov radiation, Ann. Phys. (NY) 96303.

Schwinger, J. (1970). Particles, Sources and Fields (Addison-Wesley Publ. Comp., Reading, Mass.), Vol. 1.

Schwinger, J. (1973). Particles, Sources and Fields (Addison-Wesley Publ. Comp., Reading, Mass.), Vol. 2. 